\title{
Preparation and Assessment of Nanocomposites Based on Natural Rubber Latex Reinforced With Multiwall Carbon Nanotubes for Gamma Rays Shielding Applications
}

\section{A. Abdeldaym ( $\square$ ahmedabdeldaym2000@yahoo.com )}

Egyptian Atomic Energy Authority

M. A. Elhady

Egyptian Atomic Energy Authority

\section{Research Article}

Keywords: natural rubber latex, multiwall carbon nanotubes, nanocomposites, Gamma rays, shielding

Posted Date: October 26th, 2021

DOI: https://doi.org/10.21203/rs.3.rs-1013800/v1

License: (c) (1) This work is licensed under a Creative Commons Attribution 4.0 International License. Read Full License 


\title{
Preparation and assessment of nanocomposites based on natural rubber latex reinforced
} with multiwall carbon nanotubes for Gamma rays shielding applications

\section{A. Abdeldaym ${ }^{1}$, M. A. Elhady ${ }^{2}$}

${ }^{1}$ Radiation physics department, National Center for Radiation Research and Technology (NCRRT), Egyptian Atomic Energy Authority. Cairo, Egypt

${ }^{2}$ polymer chemistry department, National Center for Radiation Research and Technology (NCRRT), Egyptian Atomic Energy Authority. P.O. 29, Ahmed El-Zomor St., El-Zohour District, Nasr City, Cairo, Egypt

Corresponding authorA.Abdeldaym

Email: ahmedabdeldaym2000@yahoo.com

\begin{abstract}
Reinforcement of the flexible shielding properties of natural rubber (NRL) was achieved through various content Multi-walled carbon nanotubes (MWCNTs) prepared from a simple solution using mixing method. Thereafter, a host of evaluative tests, using different techniques were carried to check the structural, morphological, mechanical and electrical conformity of the MWCNTs in the natural rubber matrix. More notably, the results from the x-ray diffraction (XRD), Scanning electron microscopy (SEM) and Fourier-transform infrared spectroscopy (FTIR) revealed that the nanocomposites have been successfully prepared, making them fitting to impact significant improvement on the mechanical strength of the matrix. The evaluation of the formation of MWCNT networks in the matrix, which gives insight into the nanocomposites' electrical conductivity, also showed agreeable results as the linear attenuation coefficients (l) and half-value thickness (HVT) for NRL/MWCNTs nanocomposite films were investigated . Thus, from the foregoing series of results, it can be concluded that nanocomposite films offer promising radiation-shielding properties.
\end{abstract}

Keywords: natural rubber latex, multiwall carbon nanotubes, nanocomposites, Gamma rays, shielding.

\section{Introduction}

In the field of medicine and within industry, radiation technology has been employed extensively; inescapably, radiation hazard has come to the spotlight in the world [1,2]. This has led to the development of light and flexible materials which will enable protection of workers alongside the environment [3]. This has opened up prospects for research into light-weight and flexible materials, as a means to prevent radiation across a number of applications for these types of materials [4]. An example of a material that can be employed for safe guarding purposes is the rubber materials [5]. This shielding application will help to mitigate the severity of radiation exposed to a permissible level and prevent harmful side effects to the human body [6].

At present, a diversified range of materials are being incorporated to achieve the 
preventive measure of radiation [7]. Of course, these materials each have advantages as well as disadvantages. There are numerous factors that affect the selection of these materials including; radiation category, expenditure, budget, workability and availability [8]. Of the materials used for radiation protection, the ones with the most common use are high-density rigid materials; these include materials such as concrete and lead products [9]. There are a spectrum of materials other than concrete and lead which have properties to diminish gamma and X-rays [10]. These materials also include copper, steel, tungsten, bismuth [11]. However, lead materials used for shielding have their limitations due to heaviness, low chemical and mechanical stability, high toxicity and rigidity hence, difficulty in transportation [12]. As a means to overcome the limitations of lead, scientists and researchers investigated the use of polymer composites which consist of inorganic fillers, such as micro and nanoparticles [13]. The uses of these particles bring in certain benefits to the material of shielding for radiation which improve cost, chemical stability, flexibility, and weight [14]. One such material investigated by researchers is Natural Rubber Latex (NRL). It is obtained innately from the tree named Hevea brasiliensis - considered as an industrially significant polymer .This polymer has a magnitude of applications in the fields of medicine, technology and engineering and laboratories. NRL contains non-rubber constituents such as proteins, lipids, carbohydrates and more, which is chemically denoted by the formula cis-1,4-polyisoprene $[15,16]$. NRL has several drawbacks too, which include: comparatively lower tensile modulus, lower thermal as well as oxidation stability and a higher dependence of dynamic properties of temperature because of damping which is derived from high glass transition temperature $[17,18]$. Due to these shortcomings, good compatibility of the polymer along with filler can lead to an increase in mechanical properties such as the tensile strength of the material and modulus. This is achieved by the distribution of tensile stress to the filler and polymer matrix [19]. Various commercial applications require the polymer to discharge an electrostatic charge and provide electrical conduction. MWCNTs have this property of electrical conductivity when present low content and thus, prove to be one of the ideal choices as filler [20, 21]. The goal of this research is to investigate and produce a lead-free radiation shielding material that is flexible, based on natural rubber latex and consist of varying proportions of MWCNTs. The existing process of the research is to augment the properties of NRL by rebuilding it as a matrix containing MWCNTs. With this approach, the final product will be lead-free subsequently, flexible, light-weight, resistant to fractures and at the same time have higher efficiency for radiation shielding. Moreover, we have investigated the correlation between the mechanical properties of 
nanocomposites and loading content of MWCNTs. Our research work extended to study the electrical properties of the nanocomposites and explain the results in the light of the electrical percolation theory.

\section{Experimental}

\subsection{Materials}

The natural rubber latex was used in this study is a Standard Malaysian Rubber dry rubber content $60 \mathrm{phr}$ (per hundred rubbers)). while the multi-walled carbon nanotubes (DF-1015M) were gotten from Cheil Industries, South Korea.

\subsection{Preparation of NRL/ MWCNTs nanocomposites}

A natural rubber latex (NRL) mixing method was used in preparing multi-walled carbon nanotubes (MWCNTs) filled with natural rubber latex at loading levels of 0.5 , 1.0, 1.5, and 2.0 phr. Prior to this step, reinforcement was accomplished by adding filler suspension to the natural rubber latex after which the MWCNTs were subsequently dispersed into the NRL with concurrent stirring and ultrasonication for 2 h. On the other hand, the mixture (MWCNT-filled NRL) was sonicated using ColeParmer Ultrasonic Cleaner, Heater/Digital Timer; 2.5 gal, $115 \mathrm{~V}$ for $2 \mathrm{~h}$ at room temperature in order to attain a homogenous dispersion that could enhance nanotube mechanical properties. The mixture was then cast in Teflon and left to dry at room temperature. Additional drying, after the mixture became transparent, was done in a vacuum oven in which the preparation was left for $48 \mathrm{~h}$ at $50{ }^{\circ} \mathrm{C}$. The result of this second drying was the final dry film. The nanocomposite film of NRL loaded with $0.5,1.0,1.5$, and $2.0 \mathrm{phr}$ of MWCNTs was irradiated using electron beam irradiation (EB) at $50 \mathrm{kGy}$ to vulcanize the nanocomposite film. Irradiation was carried out in air using a Cockroft Walton type electron beam accelerator (manufactured by Nissin High Voltage, Japan) at an acceleration voltage of $2 \mathrm{MeV}$ and beam current of $5 \mathrm{~mA}$. The dose rate was $25 \mathrm{kGy} /$ pass.

\subsection{Measurements}

The microstructure of NRL nanocomposite was investigated by obtaining diffraction data using XRD-6000 x-ray diffraction spectrometer manufactured by Shimadzu Corporations. This diffraction spectrometer has a copper target with a wavelength of $1.542 \mathrm{~A}^{\circ}$, and operates at a voltage of $40 \mathrm{kV}$ under an electric current of $30 \mathrm{~mA}$. The XRD patterns were consistently recorded in the $10^{\circ}-90^{\circ} 2 \theta$ angular range, and the scanning rate being 4 step $/ \mathrm{min}$.

\subsubsection{Scanning electron microscopy}

A scanning electron microscope manufactured by JEOL JSM, which operates at $30 \mathrm{kV}$, was used in investigating the surface of the samples. Leading to this examination, the surfaces had 
been pre-coated with a thin gold layer in order to minimize the instance of charging as the samples were subjected to the microscopy technique. Infrared spectroscopy measurements were also taken at ambient temperature range of $400-4000 \mathrm{~cm}^{-1}$ with the aid of a Bruker Vertex 70v vacuum FTIR spectrometer.

\subsubsection{Mechanical properties}

A model 944 KVA apparatus from Comten Industries in Pinellas Park, Florida was used in measuring the tensile of the NRL, and the standard method sustained at a cross-head speed of $50 \mathrm{~mm} / \mathrm{min}$ was followed. Samples having dumbbell shape - with a length of $50 \mathrm{~mm}$ and neck of $4 \mathrm{~mm}$ - were used in analysing tensile strength. More specifically, three of such specimens were tested and the mean values were then recorded. The flexural strength of the specimens was examined with the aid of Instron 5567 machine, with reference to the ASTM D970 standards. The Instron 5567 machine was also used in testing specimens with a dimension of $125 \times 15 \times 3 \mathrm{~mm}$. The machine was equipped with a $5 \mathrm{kN}$ load cell with a span of $100 \mathrm{~mm}$ at a cross-head speed of $50 \mathrm{~mm} / \mathrm{min}$.

DC voltage/current generator and precision digital electrometer were used for DC electrical measurements (Keithley 6515). This made it possible to identify the current produced after applying a specific voltage to the nanocomposite samples.

\subsubsection{Gamma rays shielding parameters}

The radioactive sources used in this measurement were $1-\mu \mathrm{Ci} 137 \mathrm{Cs}$ and $1-\mu \mathrm{Ci} 60 \mathrm{Co}$ point sources, which emit 662-keV and 1.25- MeV (the average of $1.173 \mathrm{MeV}$ and $1.332 \mathrm{MeV}$ ) gamma rays, respectively. The measurement was carried out inside a $6 \mathrm{~cm}$ thick lead cylinder. Samples of $15 \mathrm{~cm} \times 15 \mathrm{~cm}$ of natural rubber latex nanocomposites with thicknesses varying from $5 \mathrm{~mm}$ to $80 \mathrm{~mm}$ in $5 \mathrm{~mm}$ increments were placed between a gamma source and a gamma detector that were separated by $10 \mathrm{~cm}$. The gamma source used in this test was kept in a $3-\mathrm{cm}$ thick lead collimator with a 1-mm pinhole exit to minimize build-up effects that could increase the transmitted gamma rays caused by secondary or rescattered gamma. The NaI gamma detector (Ortec 905-4, USA) used in the measurement had a circular active area of 10 $\mathrm{cm}$ in diameter and was powered by a high voltage power supply (Canberra 3102D, USA). Its output was sent through a series of amplifiers (Ortec 590A, USA) and a counter/timer (Canberra 2071A, USA) for signal processing and counting.

\section{Results and Discussion}

\subsection{XRD analysis of the NRL/MWCNTs nanocomposites}

The purpose of upon x-ray diffraction analysis was first to discriminate the crystallographic nature of the NRL/MWCNTs nanocomposites and secondly to assess the effect of this crystallinity on the physical characteristics of the nanocomposites. For this purpose, the experiment categorised the x-ray patterns of NRL and NRL loaded with different MWCNT contents [figure 1]. The results showed the NRL film displaying similar behaviours to that of 
an amorphous polymer. A wide swelling set around $2 \Theta \sim 18^{\mathrm{O}}$ characterised this NRL film. Comparatively, the NRL loaded with different MWCNT contents showed a small peak at $22.30^{\circ}$. With the increase in the percentage of MWCNT contents, the related peak also increased relatively. The findings suggest that the increase in MWCNT content can increase the crystallinity of the prepared noncomposite samples too. The action chain defining the NRL loaded with different MWCNT contents is comprised of three key stages

At first, an increase in the percentage of MWCNT started a reaction in the molecular chains of NRL. These chains started pulling the dispersed MWCNTs into a closely packed farm. The close arrangement of the MWCNT in the cavities of the NRL further developed diffraction patterns. As a result of these patterns, the adjacent molecular chains of NRL started suffering increased pressure due to the increase in nanofiller content. The increase in nanofiller content is caused ultimately due to the increased pulling of the adjacent rubber chains

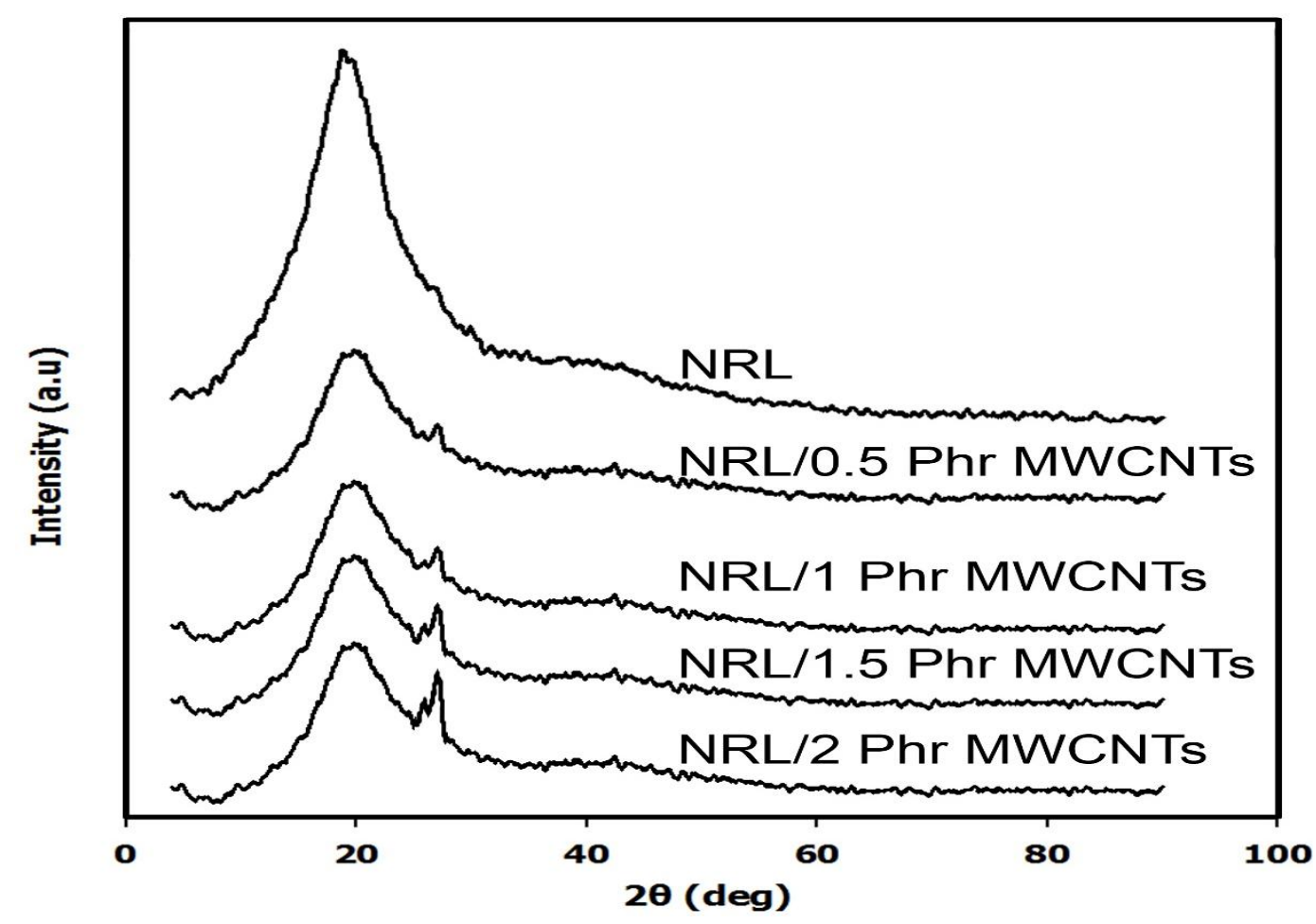

Figure 1: X Ray diffraction patterns of NRL and NRL/ MWCNTs

\subsection{Dispersion of MWCNTs in NRL nanocomposites}


The study was based upon SEM analysis. The purpose of this analysis was to exhibit the state of dispersion of MWCNTs in the NRL metrics. The rationale behind the analysis was to identify the suitable effects of such dispersion and electrical and mechanical properties of the nanocomposites. Results: The findings of the SEM analysis depicted that neat NRL generally has smooth surfaces however, rough surfaces may appear in the nanocomposites. The reason behind the occurrence of rough surfaces is the presence of the highly ordered network of nanotubes in the rubber. This network can lead to spherical granular shapes with craters and special patterns in the nanocomposite surfaces. Therefore, the surface roughness can be seen when there is an increase in carbon nanotube content. To account for the differences in the surface roughness between the nanocomposites, three aspects need to be considered. The first aspect is the interface filler/matrix phenomenon which is responsible for modifying the folding of the polymer chains. The second aspect is the inclusion of the nanofiller phase causing the differences in the thermal diffusion coefficients. The third aspect was discretional anisotropy which functions in the mobility of the polymer chains. In rubber, nanotubes are dispersed significantly due to the absence of the agglomerates and void spaces in the rubber matrix. The strong interaction between MWCNTs and rubber further increases the deep embedding of the nanotubes in the NRL. These findings report that MWCNTs can manage stress throughout the above metrics, highlighting its effective reinforcement of the properties. 


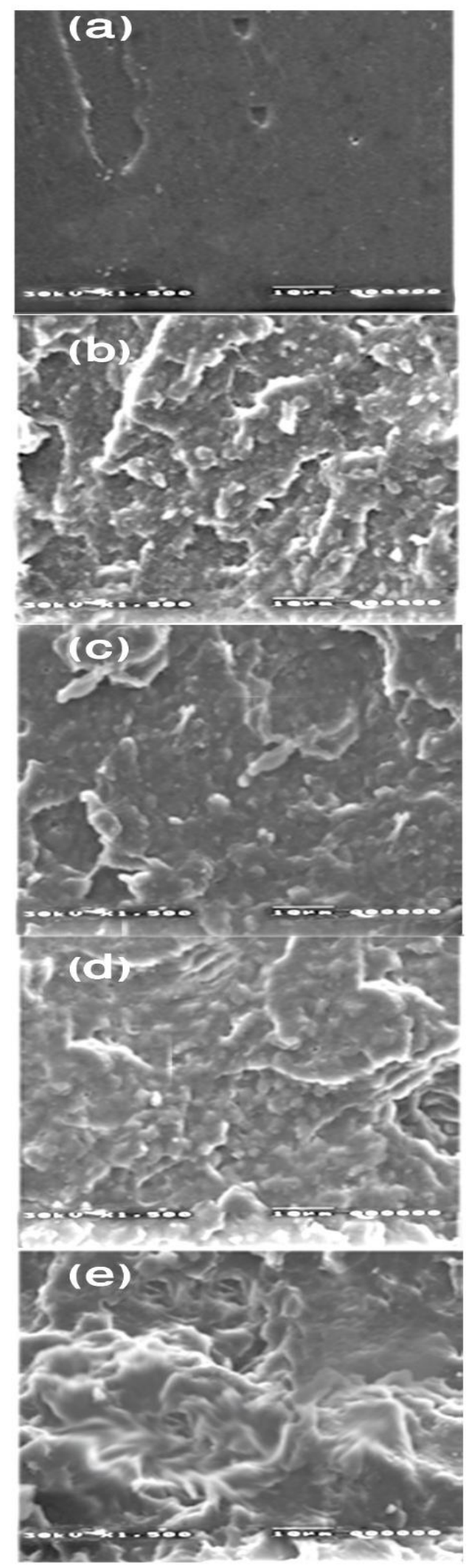

Figure 2: Surface morphology of a)NRL b) NRL/ 0.5phrMWCNTs c) NRL/ 1phrMWCNTs d) NRL/ 1.5phrMWCNTs e) NRL/2phrMWCNTs 


\subsection{Fourier Transform Infrared Spectroscopy (FTIR).}

The interactions between the functional groups of NRL and MWCNTs were examined by observing the FTIR spectra obtained from pure NRL and MWCNT-filled NRL. The samples' IR spectra are depicted in Figure 3 while their peak values are presented in Table 1. On the other hand, for the NRL sample, a wave number of 2851 indicated the presence of polyisoprene even as a variety of sharp peaks at 1517, 1441, 1241, 967 and $827=\mathrm{CH}$ stretching vibration, $\mathrm{C}=\mathrm{C}$ stretching vibration and $=\mathrm{CH}$ underwent varying degrees of alteration at $0.5,1.0,1.5$ and $2.0 \mathrm{phr}$ MWCNTs-filled NRL, with these alterations increasing proportionately in relation to the increasing percentage of MWCNTs. Furthermore, the distinctive polyisoprene absorption bands were observed with NRL/0.5 phr MWCNTs, and these (bands) included peaks at $2958 \mathrm{~cm}^{-1}, 2916 \mathrm{~cm}^{-1}$ and $2850 \mathrm{~cm}^{-1}$ which respectively corresponded to $\mathrm{CH}_{3}$ asymmetric stretching vibration, $\mathrm{CH}_{2}$ asymmetric stretching vibration and $\mathrm{CH}_{2}$ symmetric stretching vibration. Besides, $=\mathrm{CH}$ stretching vibration, $\mathrm{C}=\mathrm{C}$ stretching vibration and $=\mathrm{CH}$ out-of-plane deformation vibration were associated with peak values of $3035 \mathrm{~cm}^{-1}, 1652 \mathrm{~cm}^{-1}$ and $833 \mathrm{~cm}^{-1}$ in that (corresponding) order. Absorption bands at $1371 \mathrm{~cm}^{-1}$ and $1444 \mathrm{~cm}^{-1}$ were attributed to $\mathrm{CH}_{3}$ asymmetric deformation vibration and $\mathrm{CH}_{2}$ deformation vibration respectively. However, new absorption bands, which can be differentiated at higher loading, emerged as nanocarbon was added to the MWCNT-filled NRL matrix, with peaks of $1245 \mathrm{~cm}^{-1}$ (for $\mathrm{C}=\mathrm{C}$ stretching vibration), $1535 \mathrm{~cm}^{-1}$ (for stretching vibration of $\mathrm{C}-\mathrm{C}$ bonds of aromatic rings) and $1733 \mathrm{~cm}^{-1}$ (for $\mathrm{C}=\mathrm{O}$ stretching vibration of aromatic rings) recorded. The peaks observed from the FTIR measurements show that MWCNTs were present in the nanocomposites although MWCNT-filled NRL tends to evoke more significant changes as reflected by the increasing percentage of the filler composite. 


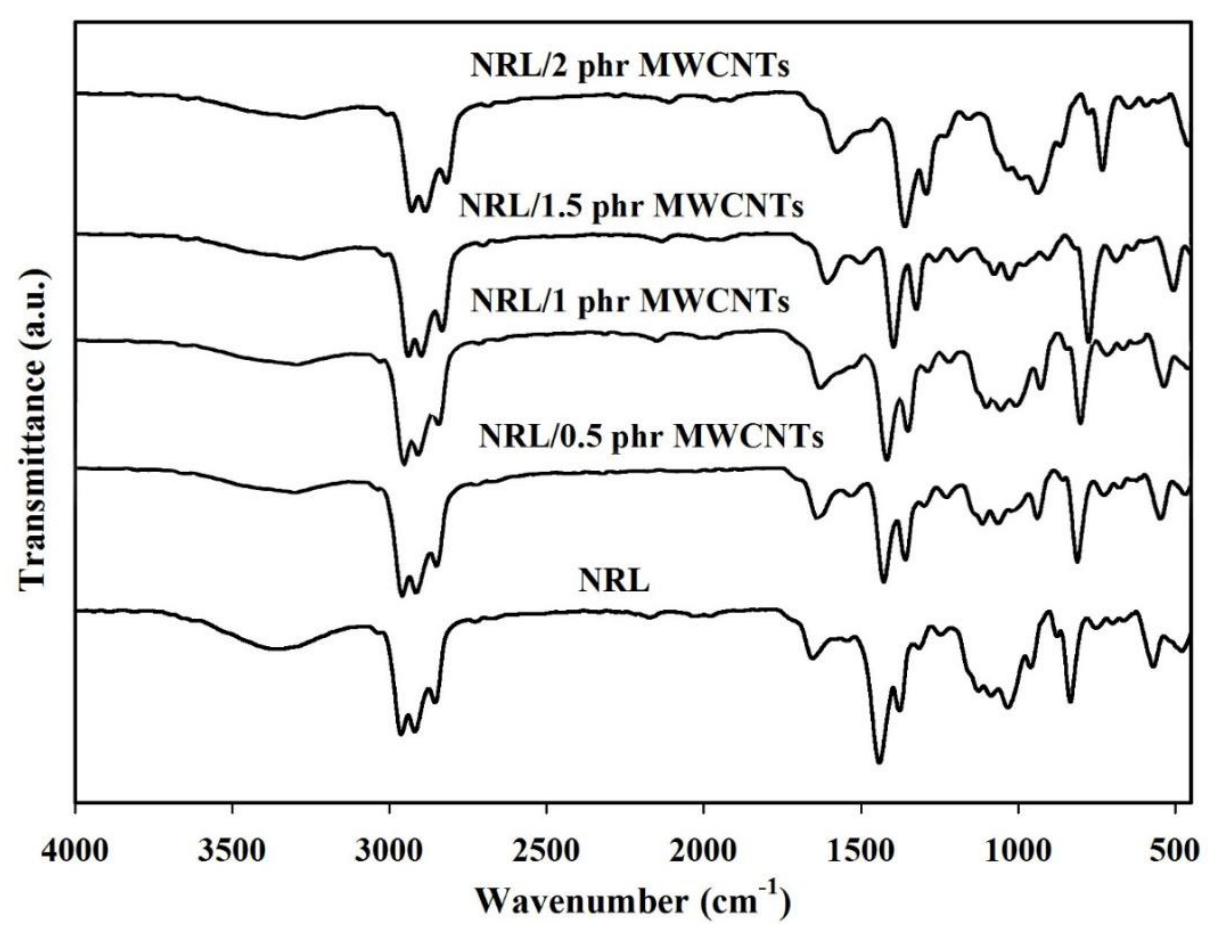

Figure 3: IR spectra of NRL and NRL/ MWCNTs

Table 1: Peak values of IR spectra of samples

\begin{tabular}{|l|l|l|}
\hline Sample & Peak frequency $\left(\mathrm{cm}^{-1}\right)$ & Bond \\
\hline & 2916,2850 & C-H stretch $\left(-\mathrm{CH}_{3}\right)$ \\
& 1597 & C=C stretching \\
Unfilled NRL & 1517,1441 & C-H bending $\left(\mathrm{CH}_{3}\right)$ \\
& 1241,1136 & C-H wag $\left(\mathrm{CH}_{2}-\mathrm{X}\right)$ \\
& $1031,965,825,753$ & $=$ C-H bend \\
\hline \multirow{3}{*}{ NRL/0.5phr MWCNTs } & 2916,2850 & C-H stretch $\left(-\mathrm{CH}_{3}\right)$ \\
& 1441,1373 & C-H bending $\left(\mathrm{CH}_{3}\right)$ \\
& $971,829,727$ & $=$ C-H bend \\
\hline NRL/1phr MWCNTs & 2916,2850 & C-H stretch $\left(-\mathrm{CH}_{3}\right)$ \\
& 1517,1376 & C-H bending $\left(\mathrm{CH}_{3}\right)$ \\
& 967 & $=$ C-H bend \\
\hline NRL/1.5phr MWCNTs & 1515,1381 & C-H stretch $\left(-\mathrm{CH}_{3}\right)$ \\
& 1235 & C-H bending $\left(\mathrm{CH}_{3}\right)$ \\
& 963 & C-H wag $\left(\mathrm{CH}_{2}-\mathrm{X}\right)$ \\
& 1507,1375 & =C-H bend \\
\hline NRL/2phr MWCNTs & 1229 & C-H bending $\left(\mathrm{CH}_{3}\right)$ \\
& 2914 & =C-H bend \\
\hline
\end{tabular}




\subsection{Mechanical properties}

For such material that is made up of rubber, the mechanical properties are considered to be significant for real as well as practical applications. NRL have stress-strain curves along with different loading content for MWCNTs, which were depicted in Fig. 4(a). The stress-strain curves comprise samples that display the same trends. It is evaluated that the level of stress mainly enhances considerably along with MWCNT. There is a good interface among the filler as well as a matrix, which is considered to be extremely important for the nanocomposites in order to uphold the stress. This is mainly due to the better interactions among NRL and MWCNTs matrix. The material by which filler is made up is transferred in MWCNTs with the stress that is produced evenly in the material of matrix mainly due to better dispersion. There is a large surface area that enables MWCNTs for even transformation that is applied over stress by the matrix as well as its ability for MWCNTs that enhances the modulus along with the stress-strain behaviour for the nanocomposites [23].

There are several reasons for the enhancements in mechanical properties by the MWCNTs in the NRL. These can be analyzed under low strains that are under $100 \%$, as several types of factors should be overcome during the process of stretching the composited of rubber that includes restoring force of rubber molecular chains, the interactions among MWCTs and NRL. Moreover, there are several interactions among the fillers-fillers that play a major role in the process. Along with the high strains that are above $100 \%$, there are interactions of the fillers with the fillers which cannot be neglected due to the breakdown of the network of fillers-fillers. The restoring force for the rubber molecular chains is considered as the main force that should be overcome. It is considered as the force that is related to the crosslink density [24].

In addition to the above information, it mainly influences the MWCNTs to alter the mechanical properties for the nanocomposites that are studied with the filler loading. The effects of increasing content of MWCNTs on tensile strength and Young's modulus of NRL nanocomposites were depicted in Fig. 4(b) and Fig. 4(c), respectively. As compared to the neat NRL, the increasing addition of MWCNTs in the nanocomposites of NRL results in increments that are remarkable in tensile strength, along with young's modulus. At the same point, there is a decline in the elongation at the breakpoint but in the tolerable range shown in Fig. 4(d). This mainly enhances the parameters which are correlated with the reinforcing effect for the MWCNTs in the NRL matrix along with inherent material characteristics for nanotubes, the degree of dispersion, aspect ratio, and interaction with the matrix of rubber. It also includes the deboning of chain segments from the surface of MWCNTs which helps in facilitating the relaxation of the segments [25]. 

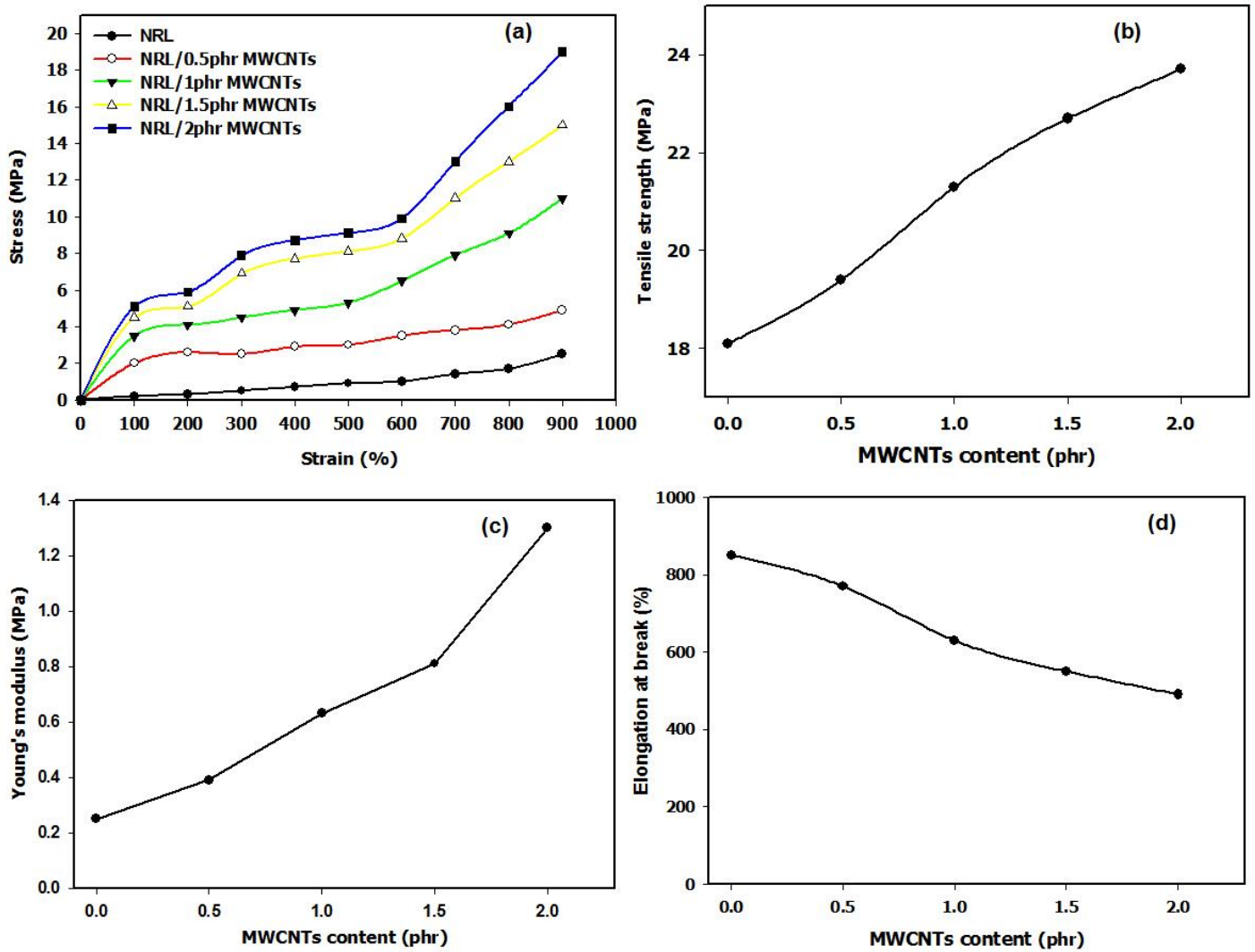

Figure 4: Mechanical properties of (a) The stress-strain curves , (b) tensile strength, (c) young modulus and (d) Elongation at break of NRL and NRL / MWCNTs nanocomposites

\subsection{Dielectric properties and electrical conductivity}

The frequency dependence of the dielectric constant, dielectric loss and AC conductivity of the NRL/MWCNTs nanocomposites at room temperature under different nanotube loading conditions is shown in Fig. 5(a, b), Fig. 6, and Fig. 7. At low frequencies, both dielectric constants and loss values are higher and then exponentially decrease with an increase in frequencies. The dielectric constant is significantly higher. When this material is exposed to electromagnetic plane waves, it produces more electric dipole moments per unit volume. As a result, the dielectric constant is high and there is dielectric loss at lower frequencies. As the frequency increases, the capability of induced dielectric polarisation decreases, resulting in a decrease in both properties. The opposite effect is seen in the material conductivity (r), where $\mathrm{r}$ increases as frequency increases. For $1 \mathrm{phr}$ MWCNTs, the value of $\mathrm{r}$ was $1.710-5 \mathrm{~S} / \mathrm{m}$ at 1 $\mathrm{kHz}$ and $4.510^{-4} \mathrm{~S} / \mathrm{m}$ at $10 \mathrm{MHz}$. This means that at higher frequencies, the external electromagnetic field induces surface currents within the material, and conduction is controlled by the conduction current, whereas at lower frequencies, displacement currents associated with generated dipole moments facilitate conduction. However, compared with the losses of electric dipole moments, the ohms losses in the conduction current are lower; hence 
the dielectric loss at low frequencies is higher. The percolation theory and the interfacial polarisation effect help explain the dielectric characteristics of nanocomposites. The conducting MWCNTs in these composites are segregated around the insulating rubber particles and can establish a variety of interfaces. The MWCNTs' huge P-orbital can create vast domains for free electrons. Interfacial polarization can occur if these electrons are directed in an electric field. Because continually rising MWCNTs become dispersed in the interfacial region, the number of interfaces rises as the MWCNT concentration increases, culminating in a completed network throughout the matrix. As a result, the dielectric constant rises as the concentration of MWCNTs rises. The chemical functionalisation can also be ascribed to the high composite dielectric constant, as the electron-withdrawing nature of carboxylic groups will exacerbate interfacial polarization.

The values of alternating current (AC) conductivity (r) at $1 \mathrm{kHz}$ for nanocomposites as a function of the fraction of multi-wall carbon nanotube (MWCNTs) volume at room temperature are shown in Figure 8 (a). The experimental conductivity (r) was modified to the well-known conductivity equation of the power law to estimate the electrical percolation threshold. If ' $\mathrm{f}$ ' is the filler volume fraction, ' $\mathrm{fc}$ ' is the percolation threshold volume fraction, and ' $\mathrm{s}$ ' is the percolation threshold volume fraction, then, for the log-log plots of the power law, the best linear fits of the conductivity data were $\mathrm{fc}=0.862$ vol per cent $(2 \mathrm{phr})$ and $\mathrm{s}=$ 4.64. The carbon nanotubes (CNTs) were positioned at the interfacial areas of the.

Fig. 8 (a) shows the value at $1 \mathrm{kHz}$, in relation to the volume of the Multi-Wall Carbon Nanopath (MWCNT) at room temperature of the alternating current (AC) conductivity ( $r$ ) values. The experimental conductivity (r) was modified to the well-known conductivity equation of the power law in order to estimate the electrical percolation threshold. These nanoscale, flexible, polymer-based composites with substantial dielectronic permittivity and dielectric loss can also be used in the low-frequency regions as electromagnetic wave absorbers. As mentioned before, the threshold of percolation is characterised by an abrupt increase of the composite conductivity throughout the entire polymer matrix by the conductive network formation of the fillers. 

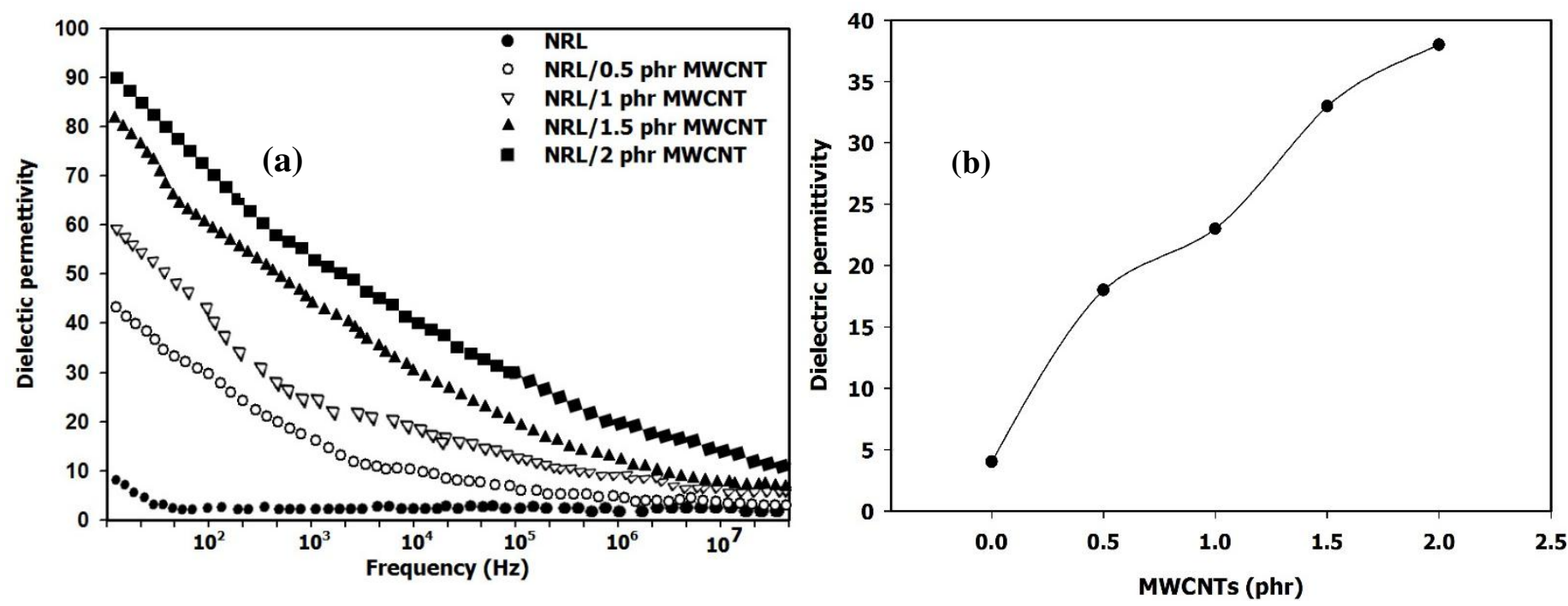

Figure 5: Dielectric permettivety as a function of frequency the NRL/MWCNTs nanocomposite

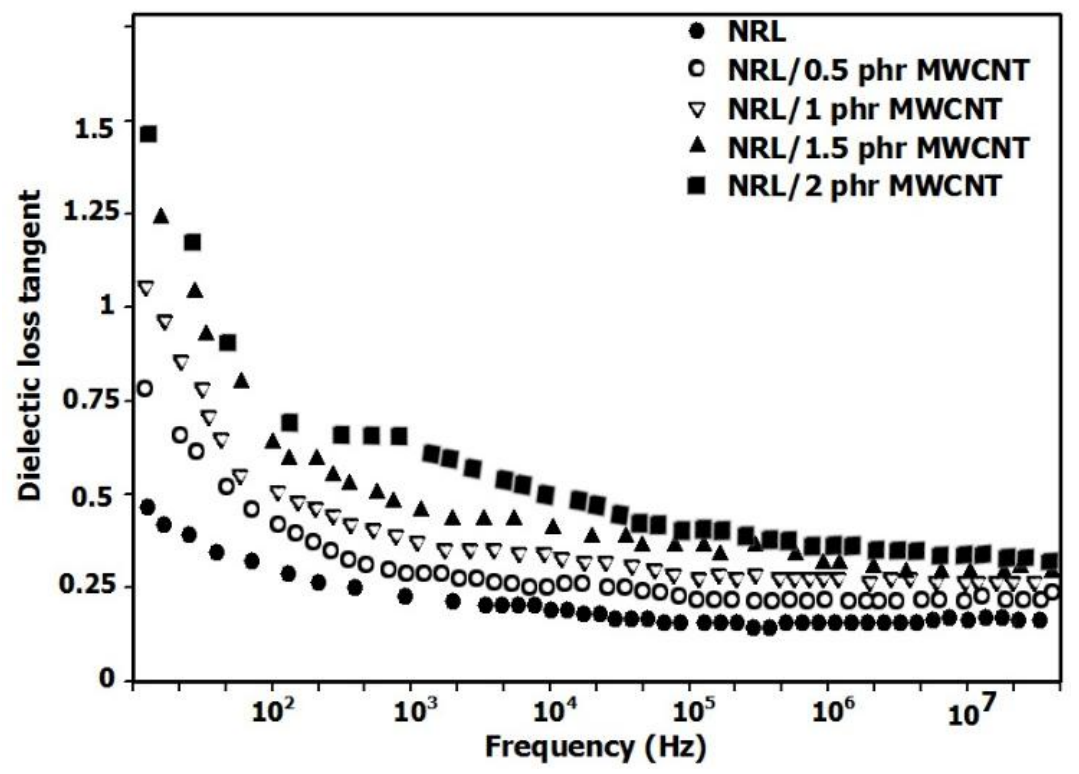

Figure 6: Dielectric loss tangent as a function of frequency of the NRL/MWCNTs nanocomposite 

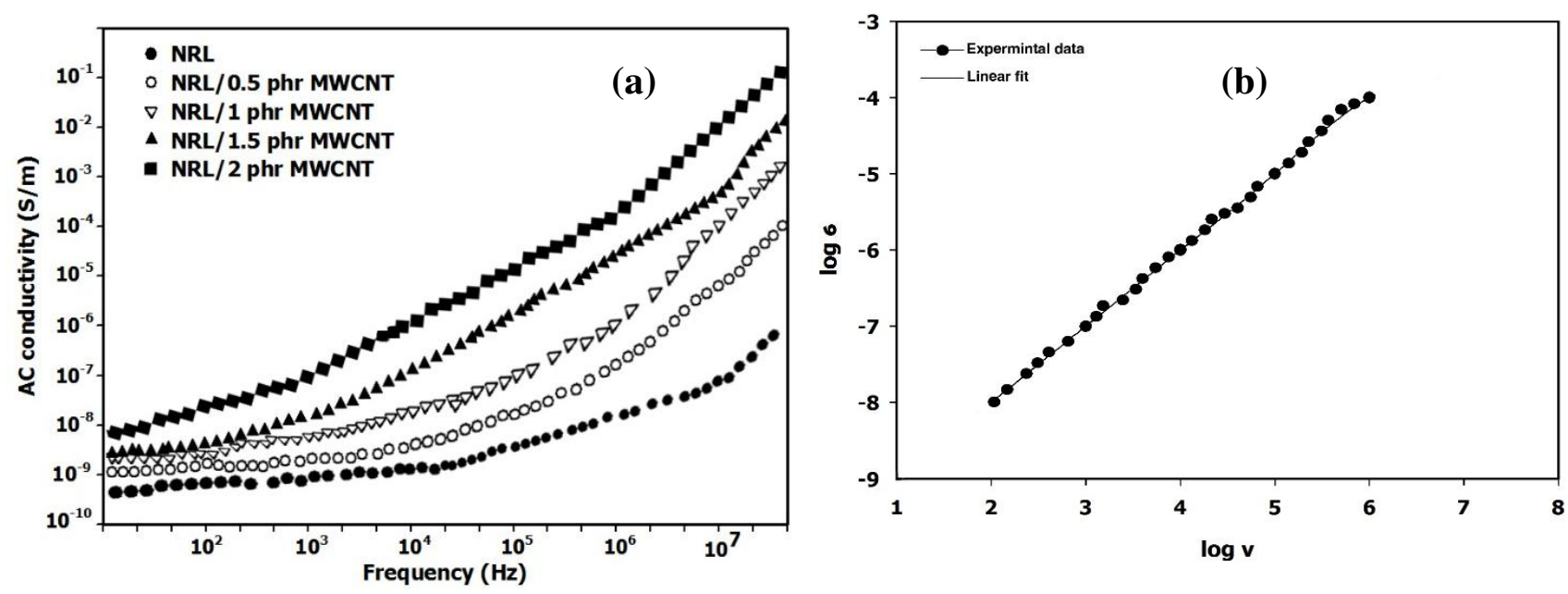

Figure 7: (a) AC conductivity (b) the best fit of the ac conductivity of the NRL/MWCNTs nanocomposite
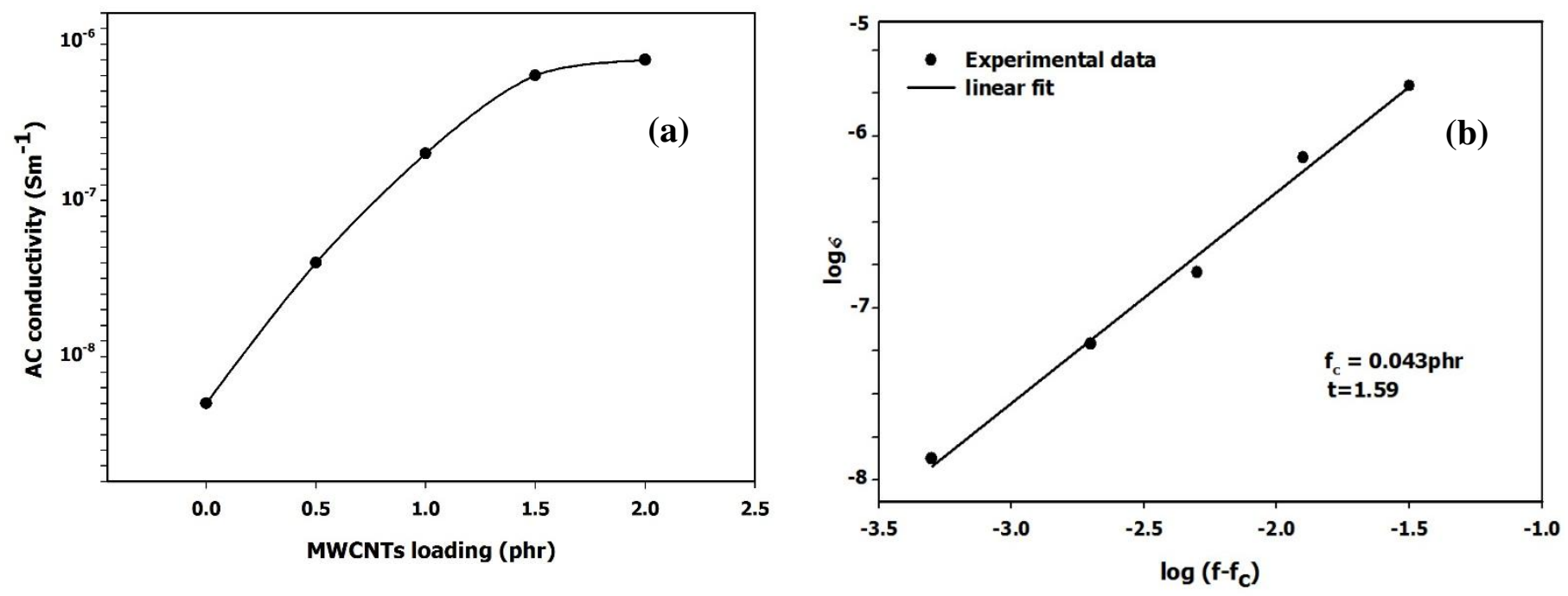

Fig. 8: (a) effect of MWCNTs on AC conductivity of NR at $1 \mathrm{kHz}$. (b) shows linear fit of the power law

\subsection{Shielding calculations}

The gamma attenuation properties of the shielding samples could be described by Eqs.

$$
\begin{gathered}
I=I_{0} \mathrm{e}^{-\mu_{I} X} \\
\mu_{I}=\mu_{m} \rho \\
H V L=\left(\ln 2 / \mu_{I}\right)
\end{gathered}
$$

Where, $\mathrm{I}, \mathrm{I}_{0}, \mu \mathrm{l}, \mu \mathrm{m}, \mathrm{HVL}$, and $\mathrm{x}$ are the intensity of transmitted gamma rays, the intensity of incident gamma rays, the linear attenuation coefficient, the mass attenuation coefficient, the half-value layer, and the thickness of the shielding material, respectively. Three independent 
1-min tests were performed and average I and I0 were recorded for each sample and thickness in order to calculate $\mu \mathrm{l}, \mu \mathrm{m}$, and HVL. The detailed definitions of $\mu \mathrm{l}, \mu \mathrm{m}$, and HVL and calculation procedures using a graph of the ratio $\mathrm{I} / \mathrm{I}_{0}$ and $\mathrm{x}$. With the increasing content of MWCNTs, the NRL/MWCNTs increased gamma protection capacity with the increased linear attenuation coefficients (l) and mass attenuation coefficients (m) and the value of half of the value layers lowered (Tables 2 and 3). (HVL). This increase was due to the growth in NRL active gamma mitigators, the amount of CNT atoms. Compared to NRL rubber composites that have the same type of gamma protective filler but differ in content, the effect of MWCNT content on gamma protection properties. Since the attenuation of gamma rays in Compton and its photoelectric effects between incident gamma rays and atoms of gamma protecting fillers is largely determined by the scattering of compost composites in Fig. 6a, the probabilities of gamma interactions were increased. Tables 2 and 3 show that the NRL loaded with 2 phr MWCNTs nanocomposites had the highest 1 and $\mathrm{m}$, as well as the lowest HVL, of all the composites, studied. This was due to improved interaction with gamma rays and greater gamma efficiency. This could be explained because higher energy gamma rays have a lower chance of material interactions than lower-energy gamma rays which lead to fewer interactions, lower transfers of energy and more gamma rays. This was fascinating since it has shown that the developed nanocomposites of NRL/MWCNTs have the comparable ability to protect gamma rays for applications requiring flexibility, durability and lightweight, such as for personal wear and/or radioactive containers. As shown in Table 3, the nanocomposites developed by NRL/MWCNTs had similar $\mu$ values to previous reports in this study. The results from these works show however that the NRL/MWCNTs have effective gamma-ray shielding capabilities and are capable, with an excellent extra characteristic in terms of flexibility and durability, of replacing hazardous plumbing materials. The correlation between gamma-ray protection and the mechanical properties of the composites understudy is another interesting result that should be discussed. Simply put, the nanocomposites developed by the NRL/MWCNTs can be used in different applications including radioactive container blinds, nuclear isolation and construction parts. The composites are also safer and more user friendly than materials with lead. 
Table 2: Linear attenuation coefficient $(\mu \mathrm{l})$ of NRL nanocomposites with additions of MWCNs for $662-\mathrm{keV}$ and $1.25-\mathrm{MeV}$ gamma rays (mean \pm standard deviation).

\begin{tabular}{|l|l|l|}
\hline \multirow{2}{*}{ MWCNs content (phr) } & \multicolumn{2}{|l|}{ Linera attenuation coeffecint; $\mu_{I}\left(\mathrm{~m}^{-1}\right)$ of NRL nanocomposite } \\
\cline { 2 - 3 } & Gamma Energy E $=622 \mathrm{keV}$ & Gamma Energy E $=1.25 \mathrm{keV}$ \\
\hline 0 & $2.1 \pm 0.3$ & $1.7 \pm 0.4$ \\
\hline 0.5 & $11.3 \pm 0.4$ & $5.7 \pm 0.3$ \\
\hline 1 & $19.5 \pm 0.7$ & $9.4 \pm 0.5$ \\
\hline 1.5 & $27.7 \pm 0.9$ & $12.1 \pm 0.8$ \\
\hline 2 & $39.6 \pm 1.1$ & $17.2 \pm 1$ \\
\hline
\end{tabular}

Table 3: Mass attenuation coefficient $(\mu \mathrm{m})$ of nanocomposites with additions of MWCNs for $662-\mathrm{keV}$ and $1.25-\mathrm{MeV}$ gamma rays (mean \pm standard deviation).

\begin{tabular}{|l|l|l|}
\hline \multirow{2}{*}{ MWCNs content (phr) } & \multicolumn{2}{|l|}{ Mass attenuation coeffecint; $\mu_{m}\left(\mathrm{X} 10^{-3} \mathrm{~m}^{2} \mathrm{~kg}\right)$ of NRL nanocomposite } \\
\cline { 2 - 3 } & Gamma Energy $\mathrm{E}=622 \mathrm{keV}$ & Gamma Energy $\mathrm{E}=1.25 \mathrm{keV}$ \\
\hline 0 & $2.2 \pm 0.3$ & $1.8 \pm 0.3$ \\
\hline 0.5 & $3.7 \pm 0.3$ & $2.1 \pm 0.3$ \\
\hline 1 & $4.3 \pm 0.3$ & $2.6 \pm 0.2$ \\
\hline 1.5 & $5.4 \pm 0.2$ & $2.9 \pm 0.2$ \\
\hline 2 & $6.1 \pm 0.3$ & $3.1 \pm 0.3$ \\
\hline
\end{tabular}

\section{Conclusion}

A host of gamma radiation shielding materials, including MWCNT-filled NRL composites with improved attenuation efficiencies were discovered in this experiment. This was well attested to by the findings from the multiple evaluation procedures carried. Firstly, the scanning electron microscopy showed the homogenous dispersion of the fillers in the matrix, even as the observations from FTIR tests revealed the successful reinforcement of MWCNTs into the NRL. Moving on to the mechanical properties; enhanced tensile strength was observed for every NRL/MWCNT nanocomposites as fracture strain reduced with increasing percentage of MWCNT contents. From the foregoing, it can be inferred that increase in the percentage of MWCNTs in rubber imposes considerable improvement on the gamma-ray shielding property of a material. Consequently, the samples prepared and tested in this investigation can serve a number of functions - from being used to cover the walls of nuclear energy centrals to functioning as a protective clothing in nuclear medicine departments and 
investigation centres, and so on - with respect to offering protection against the dangers of gamma ray.

\section{Declaration of interest}

The authors declare that they have no known competing financial interests or ... that could have appeared to influence the work reported in this paper.

\section{References}

[1] Ekwipoo Kalkornsurapranee a, Suchart Kothan ,Sirilak Intom, Jobish Johns, Siriprapa Kaewjaeng, Chittra Kedkaew, Wuttichai Chaiphaksa, Thanapong Sareein, Jakrapong Kaewkhao, Radiation Physics and Chemistry 179 (2021) 109261.

[2] Zhou R.F., Zhou X.J., Li X.B., Li P., 2016. "Radiation protection in the design of $\gamma$-ray industrial computed tomography systems," Nuclear Science and Techniques, vol. 27, 100, 2016.

[3] Keeratikarn Ninyong et al, Polymer Testing 59 (2017) 336-343.

[4] Wen Huang1, • Wenbin Yang, Quan Ma, Juying Wu, Jinghui Fan, Kai Zhang, J Radioanal Nucl Chem (2016) 309:1097-1103, J Radioanal Nucl Chem (2016) 309:1097-1103

[5] Yin Wu 1,2, Yi Cao 2,* , Ying Wu 3,* and Dichen Li, Mechanical Properties and Gamma-Ray Shielding Performance of 3D-Printed Poly-Ether-Ether-Ketone/Tungsten Composites, Materials 2020, 13, 4475; doi:10.3390/ma13204475

[6] Sondelski, B.; Nellis, G. Mass optimization of a supercritical CO2 Brayton cycle with a direct cooled nuclear reactor for space surface power. Appl. Therm. Eng. 2019, 163, 114299.

[7] Sayyed, M.I.; Lakshminarayana, G.; Kaçal, M.R.; Akman, F. Radiation protective characteristics of some selected tungstates. Radiochim. Acta 2019, 107, 349-357.

[8] Kaur, P.; Singh, D.; Singh, T. Heavy metal oxide glasses as gamma rays shielding material. Nucl. Eng. Des. 2016, 307, 364-376.

[9] Fan, J.;Wu, J.; Ma, Y. Eect of dierent size of PbWO4 particles on EPDM composite for gamma-ray shielding.Int. J. Mod. Phys. B 2020, 34, 2050046.

[10] Azreen, N.; Rashid, R.S.; Haniza, M.; Voo, Y.; Amran, Y.M. Radiation shielding of ultra-high-performance concrete with silica sand, amang and lead glass. Constr. Build. Mater. 2018, 172, 370-377. 
[11] Moharram, B.; Nagy, M.; Shaat, M.K.; Sayed, A.E.; Fayiz, M.; Dwidar, S.A.; Dorrah, M.E.; Suliman, M. Performance of lead and iron oxides nanoparticle materials on shielding properties for -rays. Radiat. Phys. Chem. 2020, 173, 108880.

[12] Chang, L. et al. Preparation and characterization of tungsten/epoxy composites for $\gamma$-rays radiation shielding. Nucl. Instruments Methods Phys. Res. Sect. B Beam Interact. with Mater. Atoms 356, 88-93 (2015).

[13] Huang, W. et al. Preparation and characterization of $\gamma$-ray radiation shielding PbWO4/EPDM composite. J. Radioanal. Nucl. Chem. 309, 1097-1103 (2016).

[14] Hayre, C.; Bungay, H.; Je_ery, C. How e_ective are lead-rubber aprons in protecting radiosensitive organs from secondary ionizing radiation? Radiography 2020, 26, e264-e269.

[15] Manasa.Ra, Sreedha Sambhudevan*a and Balakrishnan Shankar, Mechanical and Solvent Transport Properties of Nickel Ferrite Embedded Natural Rubber Nanocomposites, Materials Today: Proceedings 5 (2018) 20572-20579

[16] Suradet Matchawet1, Azizon Kaesaman1, Pornsuda Bomlai and Charoen Nakason Journal of Composite Materials 0(0) 1-12 Electrical, dielectric, and dynamic mechanical properties of conductive carbon black/epoxidized natural rubber composites

[17] S. Chuayjuljit, T. Nutchapong, O. Saravari, and A. Boonmahitthisud,"Preparation and characterization of epoxidized natural rubber and epoxidized natural rubber/carboxylated styrene butadiene rubber blends," Journal of Metals, Materials and Minerals, vol. 25, no. 1, pp. 27-36, 2015.

[18] Lih-Jiun Yu a, *, Sahrim Hj Ahmad b, Ing Kong c, Mouad A. Tarawneh d, Shamsul Bahri Bin Abd Razak e, Elango Natarajan a, Chun Kit Ang a Defence Technology xxx (xxxx) xxx Magnetic, thermal stability and dynamic mechanical properties of beta isotactic polypropylene/natural rubber blends reinforced by NiZn ferrite nanoparticles

[19] Ján Kruželák 1,*, Viera Karlíková 2, Rastislav Dosoudil 3, Katarína Tomanová 1 and Ivan Hudec 1, Reinforcement of Rubber Magnetic Composites with Zinc Salts of Acrylic and Methacrylic Acids Materials 2018, 11, 2161; doi:10.3390/ma11112161 
[20] Saurav RameshNayakaKikkeri Narasimha ShettyMohanaaMahesh Bhaskar Hegdea KamalonRajithaaAmbale Murthy MadhusudhanaaSatishkumar RNaik Functionalized multiwalled carbon nanotube/polyindole incorporated epoxy: An effective anti-corrosion coating material for mild steel Journal of Alloys and Compounds Volume 856, 5 March 2021, 158057

[21] Ricardo Bruno Pereira Negria Antonio Henrique Monteiro Fonseca Thomé da Silvaa Ana Maria Furtado de Sousab Ana Lúcia Nazarethda Silvac Elisson Brum Dutra da Rochab Improved mechanical and rheological behavior of nitrile rubber reinforced with multi-walled carbon nanotubes and carbon black dual-filler system Materials Today Communications

[22] Jobish Johns and Vijayalakshmi Rao, Characterization of Natural Rubber

Latex/Chitosan Blends International Journal of Polymer Anal. Charact., 13: 280-291, 2008

[23] Y-L. Lu, J. Ma1, T-Y. Xu, W-C. Wang, Y. Jiang3, L-Q. Zhang, eXPRESS Polymer Letters Vol.11, No.1 (2017) 21-34

[24] Maurizio Galimberti, Michele Coombs, Pierluigi Riccio, Theonis Ricco`, Simone Passera, Stefano Pandini, Lucia Conzatti, Andrea Ravasio, Incoronata Tritto, Macromol. Mater. Eng. 2012, DOI: 10.1002/mame.201200075

[25] Z. Spitalsky, D. Tasis, K. Papagelis, C. Galiotis, Prog. Polym. Sci.2010, 35, 357. 